Ferrata Storti Foundation

\title{
Sequential therapy for patients with primary refractory acute myeloid leukemia: a historical prospective analysis of the German and Israeli experience
}

Haematologica 2019

Volume 104(9):1798-1803

\section{Correspondence: \\ RON RAM \\ ronram73@gmail.com \\ Received: August 5, 2018. \\ Accepted: January 28, 2019. \\ Pre-published: February 7, 2019.}

doi:10.3324/haematol.2018.203869

Check the online version for the most updated information on this article, online supplements, and information on authorship \& disclosures: www.haematologica.org/content/104/9/1798

\section{(C)2019 Ferrata Storti Foundation}

Material published in Haematologica is covered by copyright. All rights are reserved to the Ferrata Storti Foundation. Use of published material is allowed under the following terms and conditions:

https://creativecommons.org/licenses/by-nc/4.0/legalcode. Copies of published material are allowed for personal or internal use. Sharing published material for non-commercial purposes is subject to the following conditions:

https://creativecommons.org/licenses/by-nc/4.0/legalcode, sect. 3. Reproducing and sharing published material for commercial purposes is not allowed without permission in writing from the publisher.
Ron Ram, ${ }^{1}$ Christof Scheid, ${ }^{2}$ Odelia Amit, ${ }^{1}$ Jens Markus Chemnitz, ${ }^{2}$ Yakir Moshe, ${ }^{1}$ Michael Hallek, ${ }^{2}$ Dominik Wolf, ${ }^{3}$ Irit Avivi ${ }^{1}$ and Udo Holtick ${ }^{2}$

${ }^{1}$ Bone Marrow Transplantation Unit, Tel Aviv Medical Center and Sackler Faculty of Medicine, Tel Aviv University, Tel Aviv, Israel; Department I of Internal Medicine, University of Cologne, Cologne, Germany and ${ }^{3}$ UKIM5, Medical University Innsbruck, Innsbruck, Austria

\section{ABSTRACT}

$\mathrm{P}$ rimary refractory acute myeloid leukemia (AML) is associated with a dismal prognosis. The FLAMSA-reduced intensity conditioning protocol (total body irradiation or treosulfan-based) has been described as an effective approach in patients with refractory leukemia undergoing allogeneic hematopoietic cell transplantation. A modified protocol (without amsacrine) has also recently been used. We retrospectively analyzed the transplantation characteristics and outcomes of all consecutive patients between the years 2003 and 2017 ( $n=51$ ) diagnosed with primary refractory AML who underwent transplantation at the University of Cologne and the Tel Aviv Medical Center. Median age was 54 years and median follow up was 37 months. Median time to neutrophil and platelet engraftment was 13 (range, 8-19) and 13 (range, 7-30) days, respectively. None of the patients had primary graft failure. Incidences of grade 2-4 and grade 3-4 acute graft-versus-host disease (GvHD), overall and moderate-severe chronic GvHD were 50\% (95\% CI: $41-67 \%), 12 \%(95 \% \mathrm{CI}: \quad 3-25 \%), 61 \%$ (95\% CI: $47-72 \%)$, and $42 \%$ (95\% CI: $34-51 \%$ ), respectively. Anti-thymocyte globulin administration was associated with lower incidence of acute GvHD (HR: 0.327; $P=0.02$ ). Non-relapse mortality at three months and three years were $6 \%$ and $16 \%$, respectively. Relapse incidences were $6 \%$ and $29 \%$, respectively. Overall survival rates at three months, three and five years were $90 \%$, $61 \%$, and $53 \%$, respectively. Chronic GvHD disease was associated with a decreased mortality rate (HR: 0.397; $P=0.045$ ). We conclude that sequential therapy in patients with primary refractory acute myeloid leukemia is safe and provides a remarkable anti-leukemic effect with durable survival and should be considered for every patient with primary refractory disease.

\section{Introduction}

Primary refractory acute myeloid leukemia (AML) occurs in approximately 30\% of AML patients and is a significant challenge in disease management. ${ }^{1,2}$ Prognosis is poor and the decision as to how to treat these patients depends on performance status, baseline and concomitant comorbidities, disease characteristics, and patient preference. Salvage chemotherapy, including protocols based on novel agents, results in a dismal median overall survival (OS) of 12.5 months. ${ }^{3}$

While attempts to reduce the rate of induction failure were seen to have only a modest impact, ${ }^{4.8}$ the only treatment option shown to prolong survival in patients with primary refractory disease is allogeneic hematopoietic cell transplantation (HCT). ${ }^{9,10}$ In contrast, a second conventional chemotherapy option offers almost no chance of cure for these patients. ${ }^{11,12}$ However, outcome after allogeneic HCT remains unfavorable if no response is achieved prior to transplant. ${ }^{10}$ Moreover, a substantial number of patients will never be able to undergo transplantation. 
A sequential therapy approach, based on the FLAMSA protocol was designed to overcome these obstacles. ${ }^{13-15}$ This both shortened the time to transplant (thus reducing ongoing repeated chemotherapy and neutropenia-associated side effects) and provided significant anti-leukemic activity; it results in remarkable complete remission rates and overall survival. Herein, we describe the GermanIsraeli experience using sequential protocols in patients with AML and primary induction failure, focusing on transplantation outcomes and highlighting the importance of this approach for patients with refractory AML and an available donor.

\section{Methods}

\section{Patients}

Only patients with primary refractory AML were included in this study. Patients received either FLAMSA/total body irradiation (TBI), FLAMSA/treosulfan, or FITCy conditioning regimen before HCT. Primary refractory AML was defined as unresponsiveness (at least $20 \%$ of blasts in marrow) after at least one course of $7+3$ (defined on day 28-35 marrow), four courses of hypomethylating agent, TAD/HAM, or sequential HAM-induction (s-HAM). Percentage of blasts was documented just before the start of conditioning. FLAMSA/ treosulfan instead of FLAMSA/TBI was used in patients older than 65 years or in those with significant comorbidities. ${ }^{15}$ Patients with relapsed AML were not included in this study. Details about the donor search procedures, the comprehensive treatment, and the supportive care are available in the Online Supplementary Appendix.

\section{Evaluation of response}

Engraftment was defined as the first of three days with a neutrophil count of $>0.5 \times 10^{9} / \mathrm{L}$ and a non-transfused platelet count of $>20 \times 10^{9} / \mathrm{L}$. Disease response and donor chimerism were assessed at day +30 and day +100 in peripheral blood $(\mathrm{PB})$ and bone marrow (BM). Complete remission (CR) was defined as $<5 \%$ blast cells in BM by cytomorphology and flow cytometry, and neutrophils of $>1.5 \times 10^{9} / \mathrm{L}$ in $\mathrm{PB}$. Hematologic relapse was defined by the reappearance of blast cells in the $\mathrm{PB}$, or by $>5 \%$ blast cells in BM. Death from leukemia was defined as death with refractory disease after transplantation or death from any cause after posttransplantation relapse. Non-relapse mortality (NRM) was defined as death from any cause other than refractory disease or relapse.

\section{Statistical analysis}

All patients gave informed consent to the planned treatment schedule as well as for anonymous data collection. The study was approved by the local ethics committee.

Continuous variables were described as the mean, median, standard deviation and range of number of observations, as applicable. Categorical data were described with contingency tables including frequency and percentage. Confidence intervals were calculated at two-sided $95 \%$ level of confidence. Two-sided $P<0.05$ was considered statistically significant.

Overall survival (OS) was defined as the time from HCT until the date of death from any cause. For subjects who are still alive, survival data were censored at the last known date of follow up. Disease response and disease progression were assessed according to the previously published response criteria. ${ }^{11}$

The probabilities of OS were estimated using the Kaplan-Meier method, and the log-rank test was used to evaluate the differences between groups. Probabilities of NRM were estimated with the use of cumulative incidence curves, with relapse treated as a com- peting risk. Relapse incidence was evaluated with use of cumulative incidence curves, with NRM treated as a competing risk. The Fine and Gray method was used to evaluate the differences between groups. Multivariate analysis was performed using a Cox proportional hazard regression model for OS and competing risk regression by the Fine and Gray method for NRM and relapse. We used the Duval score to stratify patient populations; patients were scored on a scale of 1-4, considering the fact that all patients were in a primary refractory state. ${ }^{16}$ SPSS version 23 , R 3.1.0 statistical software, and Prism version 5.0 were used for statistical analysis.

\section{Results}

Between March 2005 and January 2018, we identified 51 patients who had a primary refractory disease and were treated at the University of Cologne $(\mathrm{n}=30)$ or at the Tel Aviv Medical Center ( $\mathrm{n}=21$ ). Table 1 shows patients' characteristics. Median follow up of surviving patients was 37 (2-154) months. Median age was 54 (range, 18-73) years. In the majority of patients, prior chemotherapy was either TAD/HAM or sHAM ( $\mathrm{n}=30,59 \%$ ) or $7+3$ only $(\mathrm{n}=11,22 \%)$. Median time from induction chemotherapy to HCT was 84 (25-183) days. The preparative regimen was FLAMSA-TBI ( $n=13,26 \%)$, FLAMSA-Treosulfan $(n=17,33 \%)$ or FITCy-TBI $(n=21,41 \%)$. Anti-thymocyte globulin (ATG) was given as part of the conditioning in 6 of $20(30 \%)$ grafts derived from siblings and in 24 of 30 $(80 \%)$ grafts derived from an unrelated donor. In almost all cases, the graft was derived from peripheral blood stem cells.

\section{Early transplantation course}

All 51 patients were evaluated for early transplantation toxicities. Eleven patients $(22 \%)$ developed documented infections, including invasive aspergillus $(n=2,4 \%)$, gramnegative bacteremia $(n=6,12 \%)$, gram-positive bacteremia $(n=2,4 \%)$ and C. difficile-associated diarrhea $(n=1,2 \%)$. In two patients $(4 \%)$, the etiology of sepsis could not be determined.

Two (4\%) patients developed acute respiratory distress syndrome (ARDS) and two (4\%) patients developed sinusoidal obstruction syndrome (SOS). In one case, ARDS resulted in early mortality at seven days post transplantation. Data regarding mucositis were available in 21 of 51 patients $(41 \%)$. Only two $(10 \%)$ patients experienced grade $3-4$ mucositis, whereas five (24\%) did not develop any mucositis. Median time to neutrophil engraftment was 13 (range, 8-19) days and median time to platelet engraftment was 13 (range, 7-30) days. None of the patients had primary graft failure. In all patients, analyses of whole marrow chimerism showed $96-100 \%$ donorderived cells on day 30 post HCT.

\section{Graft-versus-host disease}

Median onset of grade 2-4 acute GvHD was 26 (range, 7-162) days. Among those patients who developed acute GvHD, involvement of skin, gut, and liver occurred in 17 (50\%), $14(41 \%)$, and $10(29 \%)$ of the patients, respectively. By day 100 , the cumulative incidences of grade 2-4 and grade $3-4$ acute $\mathrm{GvHD}$ were $50 \%$ (95\%CI: $41-67 \%$ ) and $12 \%$ (95\% CI: 3-25\%), respectively (Figure 1A). In univariate analysis, male gender was associated with a higher incidence of acute GvHD, while older age and ATG administration were associated with a lower incidence of 
acute GvHD. In multivariate analysis, only use of ATG had a statistically significant impact on the incidence of acute GvHD (HR 0.327, 95\% CI: 0.13-0.82; $P=0.02$ ).

Median time for the development of chronic GvHD was 9.4 (range, 2.5-12.4) months. At two years post HCT, the cumulative incidence of overall and moderate-severe chronic GvHD were 61\% (95\%CI: $47-72 \%$ ) and $42 \%$ (95\% CI: $34-51 \%$ ), respectively (Figure $1 \mathrm{~B}$ ). In univariate analysis, ATG administration was associated with lower incidence of overall chronic GvHD, while prior acute GvHD was associated with an increased incidence of chronic GvHD (Table 2). Following multivariate analysis, only prior acute GvHD was associated with a statistically significant impact on chronic GvHD (HR: 1.96, 95\% CI: 1.12-3.42; $P=0.04$ ).

Table 1. Patients' characteristcs.

\begin{tabular}{|c|c|}
\hline & All Cohort $(n=51)$ \\
\hline Domain & \\
\hline Sex, n male (\%) & $22(43 \%)$ \\
\hline Age, median, range (years) & $54,18-73$ \\
\hline Median days to HCT, range & $84(25-183)$ \\
\hline $\begin{array}{l}\text { Cytogenetics } \\
\text { Normal karyotype } \\
\text { Complex karyotype } \\
\text { Other }\end{array}$ & $\begin{array}{l}28(55 \%) \\
13(26 \%) \\
10(19 \%)\end{array}$ \\
\hline \begin{tabular}{l} 
Molecular \\
\multicolumn{1}{c}{ FLT3-ITD } \\
JAK2 \\
BCRABL \\
FLT3-TKD \\
MLL \\
RUNX1 \\
\end{tabular} & $\begin{array}{l}14(28 \%) \\
2(4 \%) \\
1(2 \%) \\
6(12 \%) \\
1(2 \%) \\
3(6 \%)\end{array}$ \\
\hline $\begin{array}{l}\text { Prior Chemotherapy } \\
\text { TAD/HAM } \\
\text { sHAM } \\
7+3 \text { only } \\
7+3 \text { plus salvage } \\
\text { Azacitidine only }\end{array}$ & $\begin{array}{c}7(14 \%) \\
23(45 \%) \\
11(22 \%) \\
8(13 \%) \\
3(6 \%)\end{array}$ \\
\hline \% blasts in marrow prior to $\mathrm{HCT}$ (range) & $24(5-93) \%$ \\
\hline $\begin{array}{l}\text { Preparative regimen } \\
\text { FLAMSA-TBI-based } \\
\text { FLAMSA-Treo-based } \\
\text { FITCy-TBI-based } \\
\text { ATG }\end{array}$ & $\begin{array}{l}13(26 \%) \\
17(33 \%) \\
21(41 \%) \\
30(59 \%)\end{array}$ \\
\hline $\begin{array}{l}\text { Donor } \\
\text { Matched-related } \\
\text { Mismatched-related } \\
\text { Matched-unrelated } \\
\text { Mismatched-unrelated } \\
\text { Female-to-male }\end{array}$ & $\begin{array}{c}19(37 \%) \\
1(2 \%) \\
26(51 \%) \\
5(10 \%) \\
12(24 \%)\end{array}$ \\
\hline $\begin{array}{l}\text { CMV status (D/R) } \\
+/+ \\
+/- \\
-/+ \\
-/-\end{array}$ & $\begin{array}{c}28(54 \%) \\
5(10 \%) \\
8(16 \%) \\
10(20 \%)\end{array}$ \\
\hline $\begin{array}{l}\text { Graft characteristics } \\
\text { Peripheral blood }(\%) \\
\text { CD34, median, range }\left(\times 10^{6} / \mathrm{kg}\right) \\
\text { CD3, median, range }\left(\times 10^{8} / \mathrm{kg}\right)\end{array}$ & $\begin{array}{c}50(98 \%) \\
6(2.6-14.6) \\
1.9(1-4.9)\end{array}$ \\
\hline
\end{tabular}

$\mathrm{N}$ : number; HCT: hematopoietic cell transplantation; TBI: total body irradiation; D/R: donor/recipient; ATG: anti-thymocyte globulin; CMV: cytomegalovirus.

\section{Non-relapse mortality}

Ten patients died because of non-relapse mortality. In six patients, death was preceded either by acute or chronic GvHD. There were three deaths within 30 days from HCT: one patient developed grade 3 acute GvHD, one patient who underwent HCT due to transformed chronic myelomonocytic leukemia (CMML) into AML with pretransplant hepato-splenomegaly developed severe SOS, and one patient developed sepsis. The cumulative incidences of NRM at 30 days, three months, one year, and three years were $6 \%, 6 \%$ and $13 \%$, and $16 \%$, respectively (Figure 2A). There were three cases of late ( $>3$ years after transplant) mortality: two patients had prior severe chronic GvHD, while the third, a 73-year old patient, died at the age of 78 with no transplant-associated toxicities. There was no difference between the three protocols in terms of NRM. Because of the low number of events, we did not perform regression analyses for NRM.

\section{Disease response and relapse incidence}

Response analysis performed on day 30 showed that only one patient ( $2 \%$ of the 48 patients who survived the

A

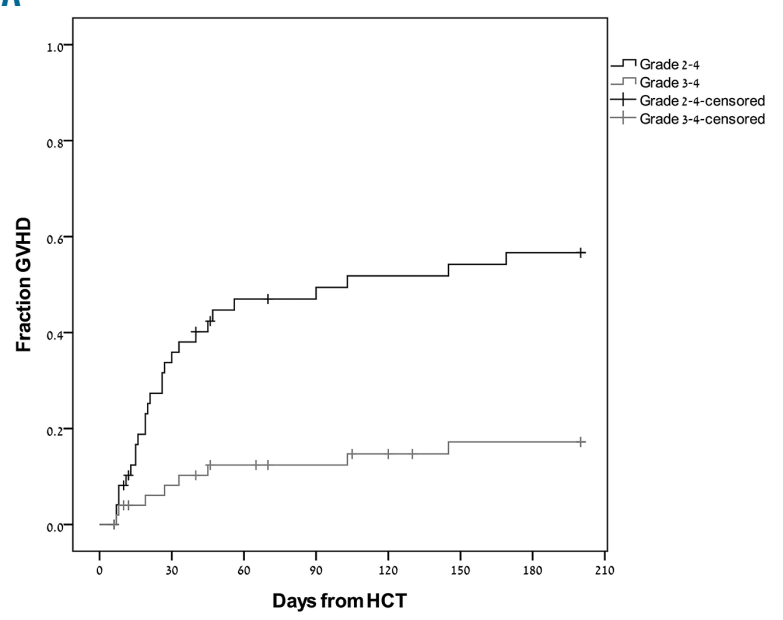

B

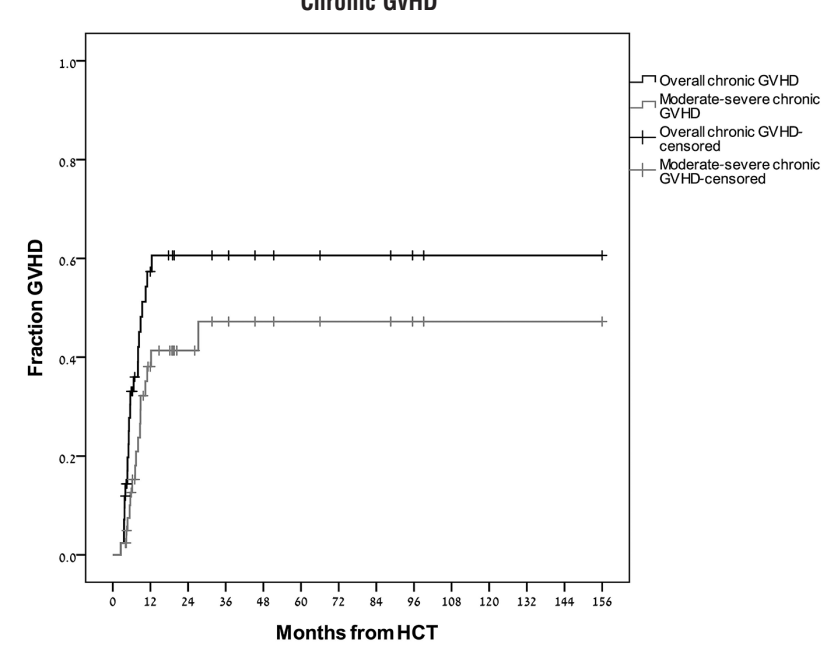

Figure 1. Incidence of graft-versus-host disease (gvHD). (A) Grade 2-4 and grade 3-4 acute GvHD. (B) Overall and moderate-severe chronic GvHD. HCT: hematopoietic cell transplantation. 
first month after HCT) did not respond to treatment, whereas all other patients obtained complete remission. There were 12 cases of relapse. Relapse incidence at three months, one year, and three years post HCT were $6 \%$, $20 \%$, and $29 \%$, respectively (Figure $2 \mathrm{~B}$ ). There were no cases of late relapses (occurring more than 3 years post HCT). Among the relapsing patients, eight (67\%) had a baseline complex karyotype and four (33\%) had FLT3-ITD with normal karyotype. There was no difference between the three protocols in terms of incidence of relapse. ATG also did not affect relapse incidence. Patients with baseline complex or monosomal karyotype had a higher risk for relapse (HR: 5.3, 95\% CI: 1.6-17.9; $P=0.008$ ). Due to the low number of events, we did not perform a full regression analysis for relapse.

Intervention at the time of relapse was donor lymphocyte infusion (DLI) $(n=5)$ and a second allogeneic HCT $(n=1)$. Only the patient who had a second transplant survived (73 months post HCT and still alive). Median time from relapse to death among all 11 patients was 1.9 months. Median time from relapse to death in patients given DLI ( $\mathrm{n}=5)$ was 5.5 months.

\section{Overall survival}

At the time of data analyses, 23 patients were alive. Incidences of OS at three months, and one, three, and five years post HCT were 90\% (95\%CI: 84-96\%), 71\% (95\% CI: $60-83 \%), 61 \%$ (95\%CI: $54-69 \%$ ), and $53 \%$ (95\% CI: 41-66\%), respectively (Figure 2C).

In univariate analyses, older age and a 9/10 mismatched graft were associated with an increase in the mortality rate, while ATG administration and chronic GvHD were associated with a decreased mortality rate (Table 2 ). In multivariate analysis, older age remained statistically significant and was associated with increased mortality (HR:
1.32, 95\%CI: 1.09-1.71; $P=0.046$ ), while only chronic GvHD was associated with decreased mortality (HR: 0.397, 95\% CI: 0.07-0.93; $P=0.045)$. Survival was not influenced by time from diagnosis to HCT as a continuous variable, even when we segregated patients into HCT $\leq 60$ days from diagnosis, HCT 61-120 days from diagnosis, and HCT $\geq 120$ days from diagnosis (median not reached, 49 months, and 35 months, respectively; $P=0.59$ ) (Figure 2D). Survival was also similar between the TBI-FLAMSA, TREO-FLAMSA, and FITCy protocols (1-year OS, 69\%, $71 \%$, and $75 \%$, respectively; $P=0.91$ )

Patients with Duval score of 4 had a mean OS of 47 (95\% CI: 12-82) months, compared to patients with a score of 2 and 3 who had a mean OS of 69 (95\% CI: 47-91) months and 61 (95\%CI: 44-78) months, respectively $(P=0.055)$.

\section{Discussion}

We summarized the long-term experience with FLAMSA-based protocols given to patients with primary refractory AML. Median age was 54 years with the oldest patient being 72 years old. Early toxicities were acceptable, with only $4 \%$ of the cases developing SOS or ARDS and only one fatal event. Incidence of severe mucositis was rare and occurred in only $10 \%$ of the patients. NRM at 30 days was $6 \%$, reflecting the feasibility of the protocol even in older patients, and highlighting the fact that, although all patients experienced prolonged and profound neutropenia, severe infections were not a significant issue.

All patients engrafted at a median of 13 days. Acute GvHD occurred in $50 \%$ of the patients and $12 \%$ developed grade 3-4 acute GvHD. These rates are quite accept-

Table 2. Univariate and multivariate analyses for transplantation outcomes.

\begin{tabular}{|c|c|c|c|c|c|c|}
\hline \multirow[t]{2}{*}{ Datum } & \multicolumn{3}{|c|}{ Univariate analysis } & \multicolumn{3}{|c|}{ Multivariate analysis } \\
\hline & HR & $95 \%$ Cl & $P$ & HR & $95 \%$ Cl & $\boldsymbol{P}$ \\
\hline \multicolumn{7}{|l|}{ Acute GvHD } \\
\hline Sex (male) & 1.41 & $1.10-5.25$ & 0.027 & 1.75 & $0.55-9.04$ & 0.13 \\
\hline Age & 0.98 & $0.95-1.00$ & 0.055 & 0.98 & 0.95-0.99 & 0.04 \\
\hline ATG & 0.39 & $0.18-0.88$ & 0.02 & 0.33 & $0.13-0.82$ & 0.02 \\
\hline Matching $(9 / 10$ vs. $10 / 10)$ & 1.16 & $0.39-3.36$ & 0.79 & & & \\
\hline Donor sex (female to male $v s$. other) & 1.38 & $0.58-3.29$ & 0.46 & & & \\
\hline \multicolumn{7}{|l|}{ Chronic GvHD } \\
\hline Sex, male & 1.2 & $0.51-2.82$ & 0.66 & & & \\
\hline Age & 0.99 & $0.97-1.01$ & 0.49 & & & \\
\hline ATG & 0.67 & $0.29-0.95$ & 0.045 & 0.79 & $0.44-1.11$ & 0.09 \\
\hline Donor sex (female to male $v s$. other) & 1.7 & $0.93-4.45$ & 0.08 & & & \\
\hline Prior acute GvHD & 1.8 & $1.23-4.7$ & 0.025 & 1.96 & $1.12-3.42$ & 0.04 \\
\hline \multicolumn{7}{|l|}{ Overall Mortality } \\
\hline Sex (male) & 1.8 & $0.76-4.26$ & 0.18 & & & \\
\hline Age & 1.48 & $1.1-1.9$ & 0.013 & 1.32 & $1.09-1.71$ & 0.046 \\
\hline Time to HCT & 1.00 & $0.98-1.02$ & 0.23 & & & \\
\hline Conditioning type & 1.22 & $0.42-3.5$ & 0.71 & & & \\
\hline ATG & 0.84 & $0.35-1.01$ & 0.05 & 0.71 & $0.22-1.17$ & 0.07 \\
\hline Matching ( $9 / 10$ vs. $10 / 10)$ & 2.25 & $1.05-6.75$ & 0.048 & 2.1 & $0.88-5.3$ & 0.090 \\
\hline Prior acute GvHD & 1.4 & $0.44-2.46$ & 0.93 & & & \\
\hline Prior chronic GvHD & 0.23 & $0.06-0.83$ & 0.025 & 0.397 & $0.07-0.93$ & 0.045 \\
\hline
\end{tabular}


able, considering the fact that $61 \%$ of the patients had an unrelated donor (12\% were only $9 / 10-H L A$ identical). Nonetheless, there were several cases of late acute GvHD. This may suggest a more cautious approach than the standard post 3-month cyclosporine-tapering schedule. Another important finding in our analysis is the fact that the administration of ATG was found to be associated with a lower incidence of acute GvHD. The impact of ATG on acute GvHD, although still controversial, was demonstrated also by others. ${ }^{17,18}$ Although the three different protocols used different doses of ATG $(15-60 \mathrm{mg} / \mathrm{kg})$, it would appear that there was no significant difference between the various doses, as has been shown previously. ${ }^{19}$ Interestingly, in our study, the use of ATG was not found to correlate with a lower incidence of chronic GvHD and was not associated with a higher relapse rate.

Non-relapse mortality at three months was low (6\%) and probably reflects the low incidence of early complications (i.e. severe mucositis and SOS) and of grade 3-4 acute GvHD. The fact that patients with primary refractory AML experience a prolonged and profound neutropenia suggest that this group may benefit from fungal and bacterial prophylaxis, as well as a weekly surveillance testing.

This protocol had significant anti-leukemia efficacy with $98 \%$ of the patients responding. Considering that, for the same population, response to salvage chemotherapy is only $30-50 \%,{ }^{20}$ (and is approaching $64 \%$ in patients receiving a direct allogeneic HCT3), our results suggest that, for selected patients, the sequential therapy approach is superior. When focusing on durable response and $O S$, in our cohort that received sequential therapy the 3-year overall survival was $61 \%$. These results are superior to previous reports of patients who had primary refractory disease, achieved complete remission after salvage chemotherapy and proceeded to allogeneic HCT (OS of $48 \%$ ), to those who were transplanted directly with a refractory disease (OS of $36 \%$ ), and those with refractory disease who never received a transplant (OS of $25 \%){ }^{3}$ Yet, although limited by the small sample size, among those patients who had post-transplant relapse, high-risk disease features were common. In addition, patients with a higher Duval score had a shorter OS compared to those with a lower score, and thus our study further validates the original Duval paper. ${ }^{16}$

In our study, the only factor that was associated with prolonged survival was prior chronic GvHD, suggesting that, although associated with significant morbidity, and sometimes also with increased mortality, in this cohort of high-risk patients, graft-versus-leukemia effect is essential.
A

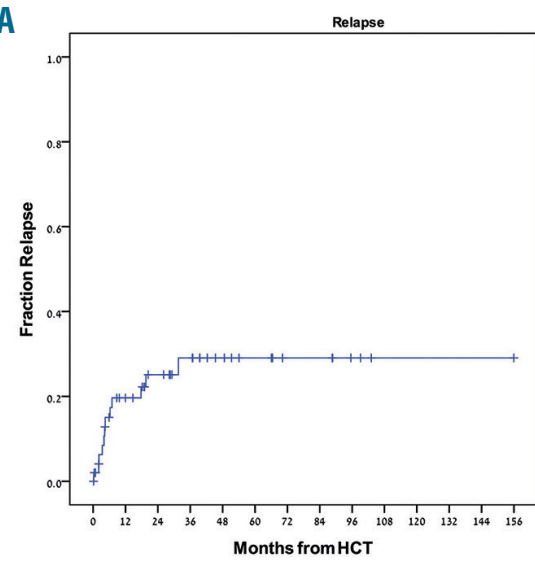

C

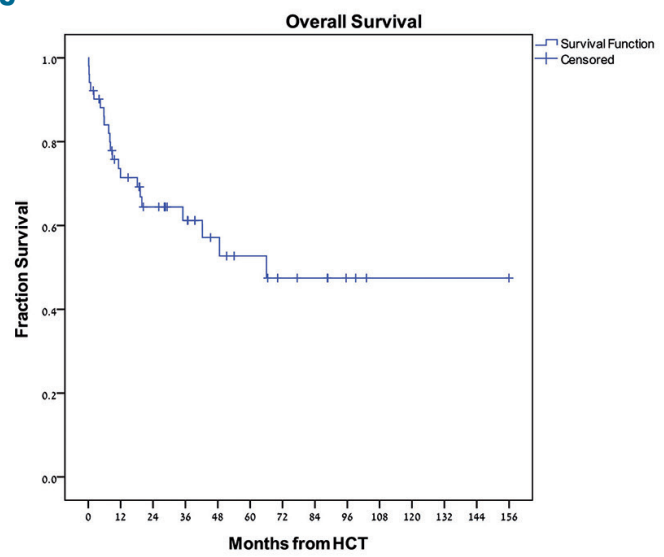

B

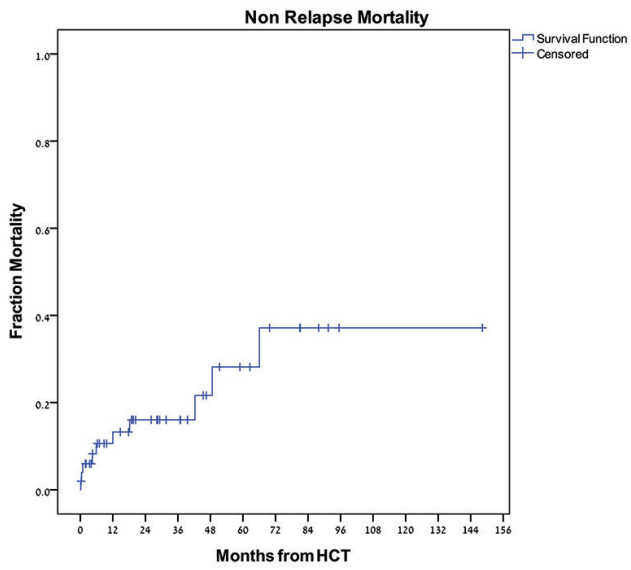

D

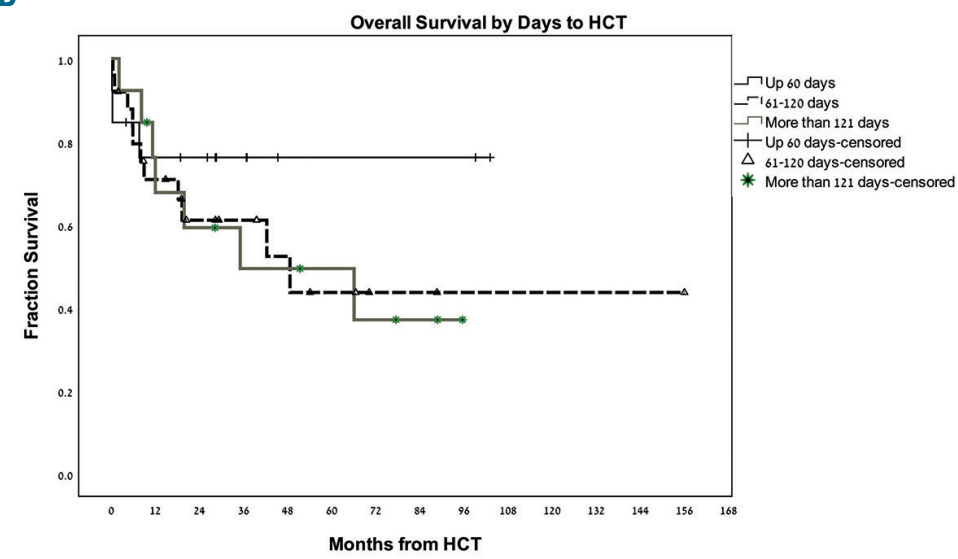

Figure 2. Transplantation outcome. (A) Relapse rate, (B) non-relapse mortality, (C) overall survival (OS), and (D) OS by time to hematopoietic cell transplantation (HCT). 
Our study has several limitations. First, as opposed to our definition of primary refractory disease, the recently published European LeukemiaNet (ELN) guidelines recommend considering primary refractory disease after only two induction cycles, irrespective of the schedule used in the second cycle. ${ }^{11}$ Nevertheless, when comparing previously published literature to our results, these recommendations do not reflect what others have considered to be standard clinical practice. Thus, although a portion of our cohort received only one cycle of induction chemotherapy and may represent a "better prognosis" group, results are still better when compared to other salvage protocols. Second, identifying patients for upfront sequential therapy may be associated with a selection bias for fitter patients. However, the median age of patients in our cohort was 54 years, which represents a 'typical' transplanted leukemia patient. Third, creating and operating a system that can quickly identify donors is challenging and requires substantial resources, which are not always available in all centers.

In summary, sequential therapy for patients with primary refractory AML is associated with a substantial antileukemic effect, relatively low toxicity, and 61\% 3-year OS. Future protocols should focus on better prevention and control of GvHD, predicting and decreasing relapse incidence in high-risk patients using minimal residual disease tests and post-allogeneic maintenance therapy (i.e. tyrosine kinase inhibitors in patients with FLT3-ITD AML), and prospectively validate these results in a larger cohort of patients.

\section{References}

1. Buchner T, Schlenk RF, Schaich M, et al. Acute Myeloid Leukemia (AML): different treatment strategies versus a common standard arm--combined prospective analysis by the German AML Intergroup. J Clin Oncol. 2012;30(29):3604-3610.

2. Juliusson G, Antunovic P, Derolf A, et al. Age and acute myeloid leukemia: real world data on decision to treat and outcomes from the Swedish Acute Leukemia Registry. Blood. 2009;113(18):4179-4187.

3. Wattad M, Weber D, Dohner K, et al. Impact of salvage regimens on response and overall survival in acute myeloid leukemia with induction failure. Leukemia. 2017;31(6): 1306-1313.

4. Lowenberg B, Ossenkoppele GJ, van Putten W, et al. High-dose daunorubicin in older patients with acute myeloid leukemia. N Engl J Med. 2009;361(13):1235-1248.

5. Fernandez HF, Sun Z, Yao X, et al. Anthracycline dose intensification in acute myeloid leukemia. N Engl J Med. 2009;361(13):1249-1259.

6. Lee JH, Joo YD, Kim H, et al. A randomized trial comparing standard versus high-dose daunorubicin induction in patients with acute myeloid leukemia. Blood. 2011;118 (14):3832-3841.

7. Schlenk RF, Frohling S, Hartmann F, et al. Phase III study of all-trans retinoic acid in previously untreated patients 61 years or older with acute myeloid leukemia. Leukemia. 2004;18(11):1798-1803.

8. Hills RK, Castaigne S, Appelbaum FR, et al. Addition of gemtuzumab ozogamicin to induction chemotherapy in adult patients with acute myeloid leukaemia: a metaanalysis of individual patient data from randomised controlled trials. Lancet Oncol. 2014;15(9):986-996.

9. Craddock C, Labopin M Pillai S, et al. Factors predicting outcome after unrelated donor stem cell transplantation in primary refractory acute myeloid leukaemia. Leukemia. 2011;25(5):808-813.

10. Schlenk RF, Dohner K, Mack S, et al. Prospective evaluation of allogeneic hematopoietic stem-cell transplantation from matched related and matched unrelated donors in younger adults with high-risk acute myeloid leukemia: German-Austrian trial AMLHD98A. J Clin Oncol. 2010;28 (30):4642-4648.

11. Dohner H, Estey E, Grimwade D, et al. Diagnosis and management of AML in adults: 2017 ELN recommendations from an international expert panel. Blood. 2017; 129(4):424-447

12. Thol F, Schlenk RF, Heuser M, et al. How I treat refractory and early relapsed acute myeloid leukemia. Blood. 2015;126(3):319327.

13. Schmid C, Schleuning M, Ledderose G, et al. Sequential regimen of chemotherapy, reduced-intensity conditioning for allogeneic stem-cell transplantation, and prophylactic donor lymphocyte transfusion in highrisk acute myeloid leukemia and myelodysplastic syndrome. J Clin Oncol. 2005; 23(24):5675-5687.

14. Chemnitz JM, von Lilienfeld-Toal $M$, Holtick $U$, et al. Intermediate intensity conditioning regimen containing FLAMSA, treosulfan, cyclophosphamide, and ATG for allogeneic stem cell transplantation in elder- ly patients with relapsed or high-risk acute myeloid leukemia. Ann Hematol. 2012; 91(1):47-55

15. Holtick U, Herling M, Pflug N, et al. Similar outcome after allogeneic stem cell transplantation with a modified FLAMSA conditioning protocol substituting 4 Gy TBI with treosulfan in an elderly population with highrisk AML. Ann Hematol. 2017;96(3):479 487

16. Duval M, Klein JP, $\mathrm{He}$ W, et al Hematopoietic stem-cell transplantation for acute leukemia in relapse or primary induction failure. J Clin Oncol 2010;28(23):37303738

17. Finke J, Bethge WA, Schmoor C, et al Standard graft-versus-host disease prophylaxis with or without anti-T-cell globulin in haematopoietic cell transplantation from matched unrelated donors: a randomised, open-label, multicentre phase 3 trial. Lancet Oncol. 2009;10(9):855-864.

18. Kroger N, Solano C, Wolschke C, et al. Antilymphocyte Globulin for Prevention of Chronic Graft-versus-Host Disease. N Engl J Med. 2016;374(1):43-53.

19. Salem G, Ruppert AS, Elder P, et al. Lower dose of antithymocyte globulin does not increase graft-versus-host disease in patients undergoing reduced-intensity conditioning allogeneic hematopoietic stem cell transplant. Leuk Lymphoma. 2015;56(4):10581065

20. Megias-Vericat JE, Martinez-Cuadron D, Sanz MA, et al. Salvage regimens using conventional chemotherapy agents for relapsed/refractory adult AML patients: a systematic literature review. Ann Hematol. 2018;97(7):1115-1153 